\title{
IMPORTANCIA DEL TRABAJO SOCIAL EN LA INTERVENCIÓN SOCIO- EDUCATIVA DE LA EDUCACIÓN SEXUAL
}

\author{
Sandra QUIROZ A. ${ }^{1}$ \\ Paula SEPÚLVEDA Q. ${ }^{2}$
}

Resumen: En el contexto educativo la figura del Trabajo Social, se encuentra en un proceso de maduración escasamente reconocido, principalmente en cuanto a sus funciones y quehacer profesional. Actualmente en el ámbito Escolar el Trabajador Social se encuentra desempeñando su labor en equipos interdisciplinarios conformados por pedagogos, psicólogos y otros profesionales asociados al área educacional, desarrollando muchas veces acciones de manera pasiva y silenciosa. En la búsqueda de las definiciones otorgadas por la FITS (Federación Internacional de Trabajadores Sociales), se señala que a través de las instituciones educativas es posible identificar problemáticas a nivel individual, familiar y comunitaria, considerando a esta unidad educativa como fuente de riqueza para la intervención, además de generar espacios para la promoción y prevención de las problemáticas sociales. El ámbito escolar es una de las áreas que permite trabajar de manera colaborativa con los directores y equipo de gestión para articular las líneas de acción que son necesarias para solucionar cualquier problema que se presente, en este sentido el Trabajador Social debe orientar, prevenir y rehabilitar, como especificidad de su profesión, además de reconocer aquellos cambios Bio-psico-sociales que desarrollan cada uno de los estudiantes y alumnos que conforman esta unidad educativa, ya que durante este proceso educacional desarrollan su personalidad, aprendizajes relacionados con las habilidades sociales para desenvolverse en nuestra sociedad e interactuar con su entorno. (Niveles Micro-MacroMeso). Es por lo anterior y en relación a las necesidades que se presentan en nuestros jóvenes y estudiantes es que debemos comprender e incorporar procesos formativos que involucren temáticas atingentes a su desarrollo y que comprenden áreas relacionadas con la educación sexual, sexualidad e identidad, con la finalidad de apoyar a las familias en este descubrimiento.

Palavras-chave: Trabajo social. Equipos interdisciplinarios. Organización escolar. La educación sexual.

\section{INTRODUCCIÓN}

En el contexto educativo la figura del Trabajo Social, se encuentra en un proceso de maduración escasamente reconocido, principalmente en cuanto a sus funciones y quehacer profesional. Actualmente en el ámbito Escolar el Trabajador

\footnotetext{
${ }^{1}$ Doctorada en innovación y planificación educativa. UAH - Universidad de Alcalá. Alcalá de Henares Madrid - España. 28801 - quirozavila@gmail.com.

${ }_{2}$ Trabajadora social. UDEC - Universidad de Concepción. Concepción - Chile. 4070386 psepulvedaquiroz@gmail.com.
} 
Social se encuentra desempeñando su labor en equipos interdisciplinarios conformados por pedagogos, psicólogos y otros profesionales asociados al área educacional, desarrollando muchas veces acciones de manera pasiva y silenciosa.

En la búsqueda de las definiciones otorgadas por la FITS (Federación Internacional de Trabajadores Sociales), se señala que a través de las instituciones educativas es posible identificar problemáticas a nivel individual, familiar $\mathrm{y}$ comunitaria, considerando a esta unidad educativa como fuente de riqueza para la intervención, además de generar espacios para la promoción y prevención de las problemáticas sociales.

El ámbito escolar es una de las áreas que permite trabajar de manera colaborativa con los directores y equipo de gestión para articular las líneas de acción que son necesarias para solucionar cualquier problema que se presente, en este sentido el Trabajador Social debe orientar, prevenir y rehabilitar, como especificidad de su profesión, además de reconocer aquellos cambios Bio-psico-sociales que desarrollan cada uno de los estudiantes y alumnos que conforman esta unidad educativa, ya que durante este proceso educacional desarrollan su personalidad, aprendizajes relacionados con las habilidades sociales para desenvolverse en nuestra sociedad e interactuar con su entorno (Niveles Micro-Macro-Meso).

Es por lo anterior y en relación a las necesidades que se presentan en nuestros jóvenes y estudiantes es que debemos comprender e incorporar procesos formativos que involucren temáticas atingentes a su desarrollo y que comprenden áreas relacionadas con la educación sexual, sexualidad e identidad, con la finalidad de apoyar a las familias en este descubrimiento.

Partiendo desde la base que La sexualidad es una parte orgánica del lenguaje de la vida, de su creación y recreación inagotables, del ser y devenir de la especie humana, de cada hombre y mujer dotados de estructuras anatómicas y de posibilidades fisiológicas para realizar una función sexual, está ligada al placer, al descubrimiento, al desarrollo personal, a lo interaccional, a lo lúdico, a lo comunicacional, a lo nuevo, a la magia, a la belleza, a la naturaleza, a la creación, a la salud y al amor (CASTELLANOS; GONZALEZ, 1996).

Antecedentes: Educación sexual en Chile, durante el año 1960 se promulgó la "ley de Planificación familiar" 
El ministerio de Educación de Chile en su página Web, contempla las bases y la fundamentación de la educación sexual definiéndose por medio de lo que pensamos, de lo que sentimos y de cómo actuamos.

Centrar la atención del estudiante en lasconducta que promuevan el autocuidado en la salud mental y física, relacionarse con otros en un medio de respeto, tener la capacidad de tomar correctas decisiones en la vida frente a los acercamientos inapropiados por medios tecnológicos.

Con el fin de fundamentar y ampliar el área de intervención se entregan un mayor conocimiento sobre el tema con las Orientaciones Técnicas Internacionales sobre Educación en Sexualidad, materia que menciona en sí:

\section{- $\quad$ Reducir la información incorrecta}

- $\quad$ Aumentar el conocimiento y manejo de información correcta

- Clarificar y consolidar valores y actitudes positivas

- Fortalecer las competencias necesarias para tomar decisiones fundamentadas y la capacidad de actuar en función de ellas

- $\quad$ Mejorar las percepciones acerca de los grupos de pares y las normas sociales

- Aumentar y mejorar la comunicación con padres, madres y otros adultos de confianza.

Además señala que el sector de educación juega un importante papel en la preparación de niños, niñas y jóvenes para asumir roles y responsabilidades propias del mundo adulto (UNESCO, 1996). La transición hacia la adultez requiere estar informado y equipado con competencias y conocimientos que permitan elegir alternativas responsables tanto en nuestra vida social como sexual. Adicionalmente, en muchos países las personas jóvenes viven su primera experiencia sexual mientras aún asisten a la escuela, hecho que confiere a este entorno aún más valor en cuanto a representar una oportunidad para impartir educación acerca de la salud sexual y reproductiva.

Una educación en sexualidad efectiva puede entregar a las personas jóvenes información culturalmente relevante, cinéticamente rigurosa y apropiada a la edad del estudiante. Ésta debe incluir oportunidades estructuradas que les permitan explorar sus valores y actitudes, poniendo en práctica competencias esenciales para la toma de decisiones para elegir con fundamento la forma que desea conducir su vida sexual (CHILE, s.d.). 


\section{Marco teórico}

Nuestro Marco teórico se sustenta en aquellos aspectos que sustentan nuestra investigación y que están directamente relacionados con la temática abordada.

\section{Teoría Ecológica: Bronfenbrenner}

Bronfenbrenner, Psicólogo Estadounidense en 1987 propone la teoría ecológica del desarrollo de la conducta humana, es un modelo integrador, que engloba a modelos como el biológico, psiquiátrico, psicosocial, y sociocultural.

Como marco teórico establece que cada uno de los seres humanos posee estos cuatro niveles, según Bronfenbrenner (1987) estos sistemas personales son compartidos a través de mecanismos de interrelaciones entre los individuos y una serie de factores externos.

El modelo Ecológico ha sido uno de los modelos más importantes para la explicación de la influencia que posee el contexto en las conductas del individuo y su desarrollo humano. Es importante señalar que dentro de los aportes obtenidos se encuentra la evolución de los individuos entendido como un proceso de carácter dinámico y recíproco, Bronfenbrenner (1987) explica el ambiente social al cual pertenecemos como "[...] una organización anidada de cuatro sistemas concéntricos, estableciendo interacciones que fluyen hacia atrás y hacia delante entre los cuatro sistemas”. (CRAIG, 2001 apud PERDOMO ARCIA, 2014).

Este modelo postula que cada individuo pertenece a estos sistemas ubicando desde una perspectiva céntrica a las personas en interacción con diferentes sistemas, en contextos inmediatos y remotos, los cuales interactúan entre sí y con los individuos durante su desarrollo, esta visión ecológica destaca también la preponderancia de los ambientes en que nos desplazamos, considerándolos como imprescindibles, estos escenarios definirán un desarrollo como una transformación perdurable, desde la perspectiva en que la persona percibe su mismo entorno y el ambiente que lo rodea de manera extensa.

Este autor, propone para la comprensión de la sociedad cuatro sistemas, que pueden influir en la problemática a investigar, estos son:

- $\quad$ Nivel Microsistema: es “[...] el patrón de actividades, roles y relaciones interpersonales que la persona en desarrollo experimenta en un entorno determinado, 
con características físicas y materiales particulares.” (BRONFENBRENNER, 1987, p.231). En el individuo se analizan aspectos cognitivos, conductuales, interacciónales y psicodinámicos, como lo son historia de vida, características de la personalidad, baja instrucción educacional, bajos ingresos, inestabilidad psicológica, estilo de resolución de conflictos, creencias y valores internalizados.

- Nivel Mesosistema: “[...] conjunto de interrelaciones de dos o más entornos en los que la persona en desarrollo participa activamente." (BRONFENBRENNER, 1987, p.231). Focaliza en las relaciones interpersonales que se dan en características físicas y materiales determinadas. En este sistema se incluye al individuo, la familia y las redes sociales primarias.

En relación a la familia los elementos estructurales de esta son importantes ya que se aprenden patrones y conductas, que propicien o no la educación sexual, además de considerar que las redes sociales primarias contribuyen y han cobrado mayor importancia en los jóvenes, por lo que se han convertido en el principal método de consulta y temas de actualidad.

- Nivel Exosistémico: “[...] uno o dos entornos que no incluyen a la persona en desarrollo como participante activo, pero en los cuáles se producen hechos que afectan a lo que ocurre en el entorno que comprende a la persona en desarrollo, o que se ven afectados por lo que ocurre en ese entorno". (BRONFENBRENNER, 1987, p.233).

Incluye a las instituciones sociales y grupos que coexisten en la sociedad, en estos se incluyen al sistema educacional, sistema judicial, sistema de salud, sistema policíaco, ámbito laboral, ámbito de ocio y recreación, medios de comunicación masiva, organizaciones religiosas, organizaciones gubernamentales y no gubernamentales.

- $\quad$ Nivel Macrosistémico:

[...] se refiere a las correspondencias, en forma y contenido, de los sistemas de menor orden (micro-, meso- y exo-) que existen o podrían existir, al nivel de la subcultura o de la cultura en su totalidad, junto con cualquier sistema de creencias o ideología que sustente estas correspondencias". (BRONFENBRENNER, 1987, p.281).

Están incluidas las estructuras de las sociedad a través de la creencias y los valores culturales, como la concepción de poder, regímenes de poder; patriarcado o matriarcado, concepción de roles para hombres y mujeres, el uso de la fuerza y el abuso, 
ideas de igualdad y desigualdad de género.

Se resaltan los siguientes aspectos; el concebir a la persona como un ser que no solamente repercute en el ambiente, sino que por el contrario se encuentra en constante evolución de manera progresiva y desarrollo produciéndose una interacción creciente entre el ambiente e influyendo recíprocamente, por esto se menciona en su modelo una acomodación mutua entre las personas y su entorno, siendo bidireccional y considerando estos niveles como estructuras concéntricas en donde cada una contiene a la siguiente.

La siguiente información representa el resultado de las opiniones de los apoderados que declaran su nivel de información en relación a la Educación Sexual que proporciona a sus hijos, esta encuesta se desarrolló en una reunión de apoderados del nivel séptimo y octavo años que corresponde a edades de estudiantes entre 11 y 15 años .

\section{Resultados}

Grafico 1 - ¿Qué entiende por Educación Sexual?

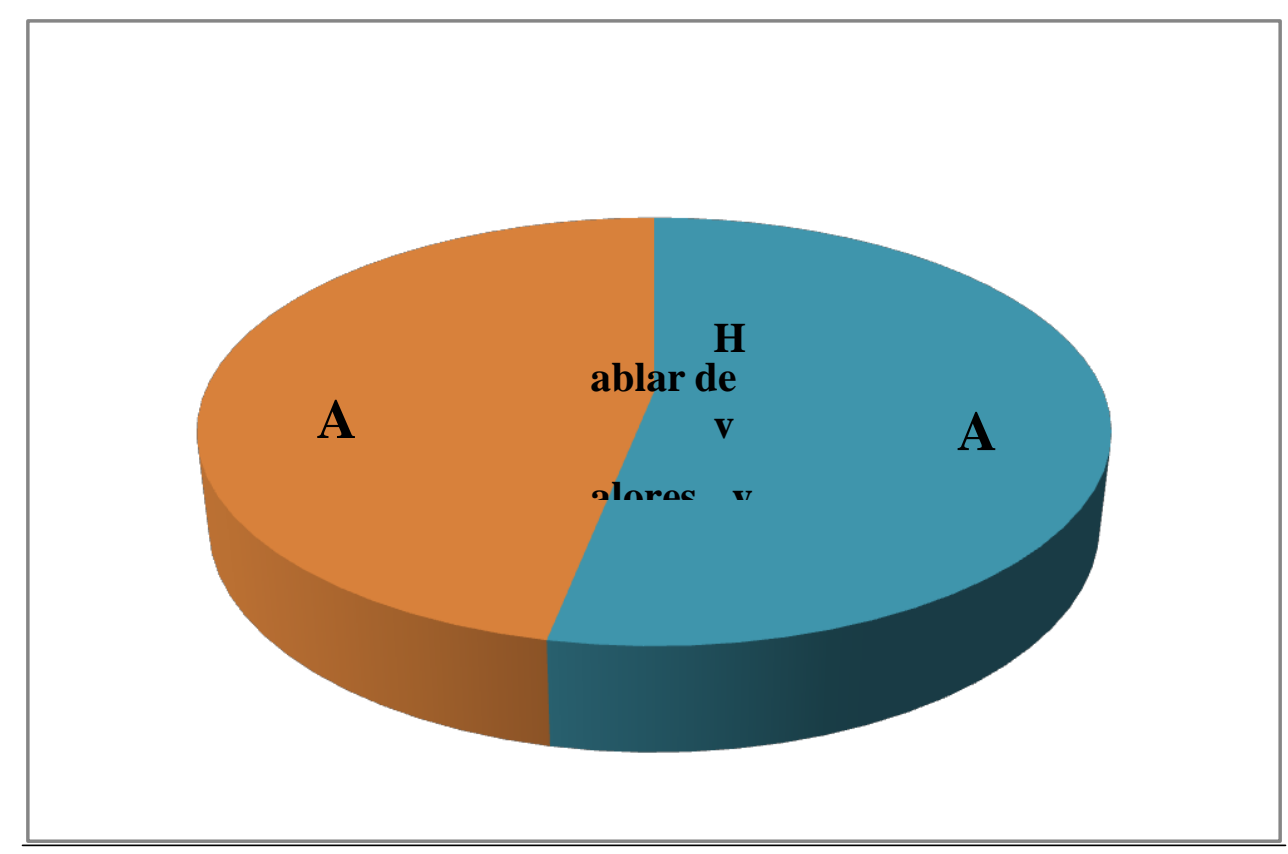

Fuente: Elaboracción propia.

De acuerdo a los resultados obtenidos es posible apreciar que a partir de la muestra perteneciente al curso $8^{\mathrm{a}}$, es posible identificar que un $47 \%$, considera que la 
educación sexual consiste en hablar de valores y relaciones de pareja, de la misma manera la muestra correspondiente al $7^{\mathrm{a}}$, considera el mismo concepto en un 53\%.

Con una frecuencia correspondiente a 25 apoderados, identifican que para ellos la educación sexual es conversar con sus hijos de anticoncepción, embarazo y enfermedades de transmisión sexual.

Grafico 2 - ¿A quien le corresponde Principalmente hablar de sexualidad con un Adolescente?

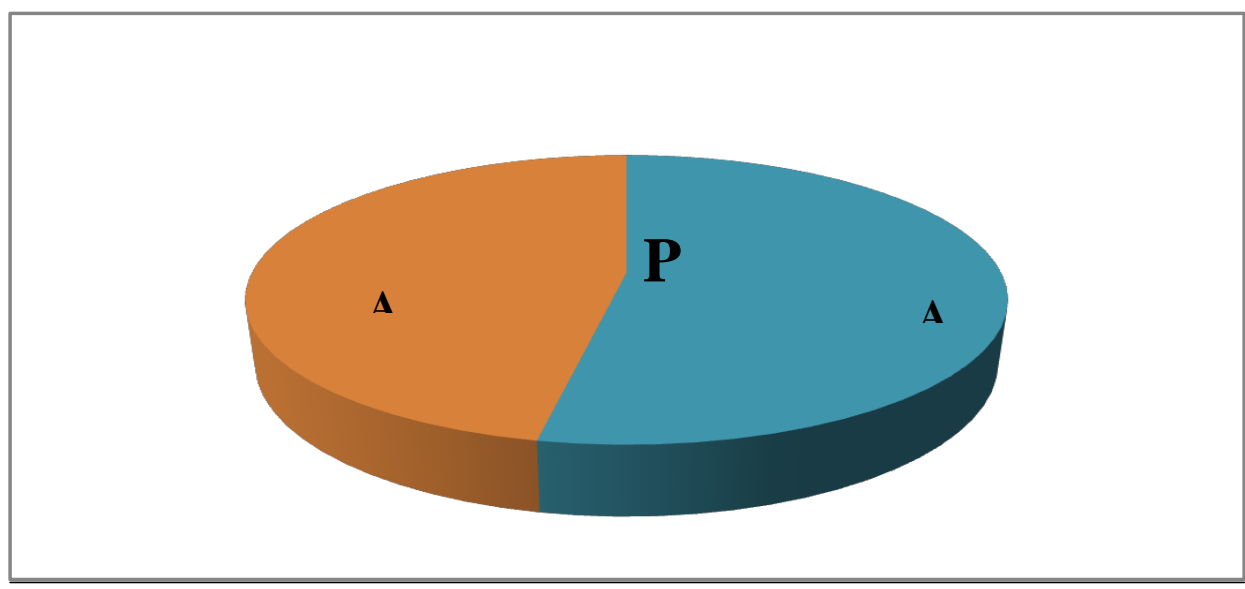

Fuente: Elaboracción propia.

Apoderados identifican que quienes son los encargados de hablar sobre estos temas relacionados con la sexualidad son principalmente los padres, identificando que está educación debería partir principalmente desde el hogar, en segundo lugar se posiciona el establecimiento educacional con la finalidad de colaborar y apoyarlos en esta tarea.

De acuerdo a la teoría anteriormente expuesta es posible identificar que en el nivel meso sistémico (familia), padres identifican que es en este núcleo en el cual se debe dialogar en relación a la sexualidad con el adolescente, lo cual se considera un nicho para el Trabajador Social, en sus intervenciones familiares orientando principalmente a los padres, sobre cómo se deben abordar estas problemáticas y articulando también las redes que apoyen al grupo familiar en relación a la sexualidad, además de considerar la importancia y el apoyo que debe recibir la familia desde las redes comunitarias de su sector (sistema, centros de salud, establecimientos educacionales, etc) 
Grafico 3 - Relevancia de la participación de la trabajadora social en la Educación Sexual de los Colegios

\section{Trabajadora social Y Educación Sexual en los Colegios}

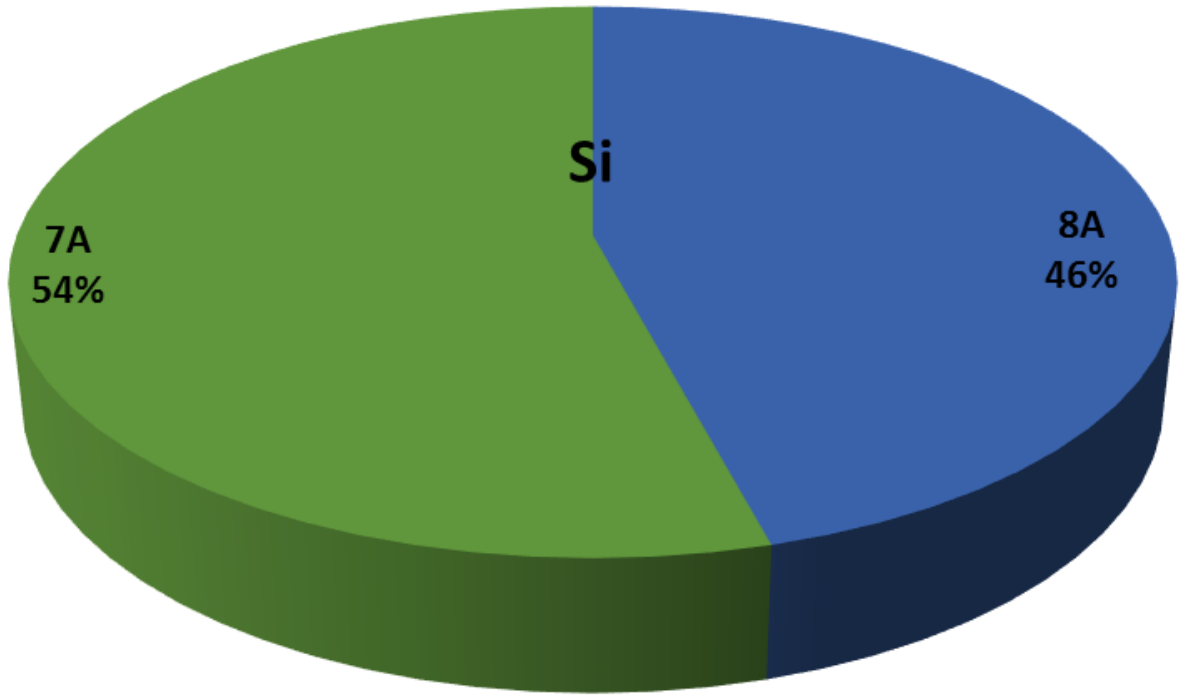

Fuente: Elaboracción propia.

En relación a la pregunta correspondiente a la relevancia que debe poseer la Trabajadora Social en la educación sexual de los colegios, es posible identificar que apoderados del $7^{\mathrm{a}}$ señalan en un $54 \%$ que es importante, y apoderados del $8^{\mathrm{a}}$, en un $46 \%$, por lo cual se considera de vital importancia potenciar a este profesional en su participación en torno a la educación sexual de manera de orientar y brindar sus herramientas junto al equipo del establecimiento profesional. 
Grafico 4 - Importancia de la visita domiciliaría de la trabajadora Social para orientar a las familias en la Educación Sexual

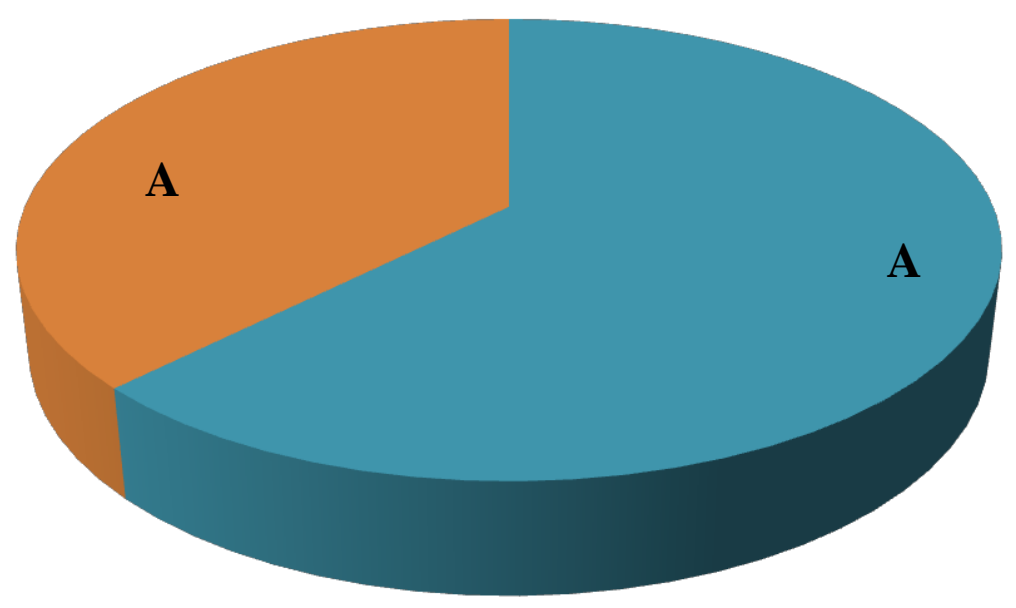

Fuente: Elaboración propia.

En relación al ítem, importancia de la visita domiciliaria para orientar a las familias en Educación sexual, es posible identificar que en la muestra correspondiente al $7^{\mathrm{a}}$, correspondiente a un total de 18 apoderados, 8 responden que sí y 10 no le asignan valor, por otro lado en la muestra del $8^{\mathrm{a}}$, de un total de 14 apoderados, 8 responden que sí y un total de 6 , responden que no es importante la visita domiciliaria para fomentar la educación sexual, dando cuenta que los padres presentan dificultades para identificar la importancia de esta técnica privativa del Trabajo Social, considerando que a partir de ella es posible identificar, estudiar y observar el ambiente social en la cual se encuentra la familia inserta, proporcionar información (en relación a la educación sexual), además de permitir diagnosticaralguna otra problemática que afecte al alumno, otorgando apoyo a la familia y al estudiante en su propio hogar, puesto que la falta de educación sexual juega un rol importante, situación que puede ser visibilizada a través de la "Visita domiciliaria".

\section{Conclusiones}

De acuerdo a la recopilación de información obtenida es posible señalar que padres necesitan capacitación para entender el desarrollo de la sexualidad en sus niños, niñas y adolescentes, en general comprender el concepto de "educación sexual”, como 
el hablar acerca de valores, amor y relaciones de pareja, además de asignarle en segundo lugar importancia a la anticoncepción, embarazo y enfermedades de transmisión sexual, consideran las dificultades que presentan para abordar el tema, en ambos cursos a los cuales fueron encuestados señalando que no logran la atención de sus hijos, además de en segundo lugar mencionar que no saben cómo abordar el tema, lo cual da cuenta de dificultades en la comunicación con sus hijos y de la falta de herramientas para tratar la temática en el sistema familiar, otro antecedente relevante es que los apoderados reconocen que los "jóvenes no hablan de sexualidad con sus padres, debido a que sienten vergüenza”.

En relación al rol del Trabajo Social en la educación sexual es posible identificar que apoderados reconocen que la orientación de esta profesión les ayudaría y les permitiría abordar esta temática, sin embargo, en relación a las visitas domiciliarias no consideran como vital esta acción para educar acerca de la educación sexual en el sistema familiar, lo cual da cuenta que no se encuentra arraigado (en este ámbito) en los apoderados las funciones que pudiera desarrollar esta profesión en el desarrollo de sus niños, niñas y adolescentes, se plantea la necesidad de trabajar en conjunto con las familias la educación sexual, como un medio para llegar a la realidad particular de cada uno de los integrantes del grupo familiar, de acuerdo al enfoque teórico mencionado anteriormente, la educación sexual plante una necesidad de comprender al niño, niña y/o adolescente en los distintos sistemas en los cuales desenvuelve, en este sentido la sexualidad trasciende lo biológico, las interacciones sociales, el sistema familiar, etc.

La educación sexual es un proceso, no sólo puede ir ligada a un programa de educación o a una institución específica, sino que más bien a un trabajo colaborativo en los diversos sistemas en los cuales se desenvuelve nuestro sujeto de atención. 
Abstract: In education the figure of Social Services, is in a process of maturationrecognized, especially in terms of functions and professional work. Currently in the school social worker is carrying out his work in interdisciplinary teams of teachers, psychologists and other related educational field professionals, the development of actions, often passively and quietly. In search of the definitions given by the FITS (International Federation of Social Workers) said that through educational institutions can identify problems at individual, household and community level, considering this educational unit as a source of wealth for intervention and create opportunities for promotion and prevention social problems. The school environment is an area that can work in collaboration with the directors and management team to articulate the lines of action that are necessary to deal with any problems. That may arise in this area should guide the social worker, prevent and rehabilitate as specificity of their profession and recognize these bio-psycho-social changes that develop students and students who make up this educational unit, as during this educational process to develop their personality, learning social skills related to work in our society and interact with their environment. (Levels micro-meso-macro). It is for this and needs that arise in our youth and students is that we understand and incorporate processes involving atingentes for learning development issues and includes areas related to sex education, sexuality and identity to support families in this discovery.

Key words: Social work. Interdisciplinary team. School organization. Sex education.

\section{REFERÊNCIAS}

BRONFENBREMNER, U. La ecología del desarrollo humano. España: Editorial Paidós, 1987.

CASTEllanos, B.; GOnZÁleZ, A. Sexualidad y Géneros. Tomo I. Bogotá: Cooperativa Editorial Magisterio, 1996.

CHILE. Ministerio de Educación de Chile. Mineduc. [Online]. [S.d.]. Disponible en: <http://mineduc.cl>. Acceso en: 21 ene. 2015.

PERDOMO ARCIA, M. X. Intervención psicosocial en la comunidad. Fase III Implementación de trabajo. Ibague: Universidad Nacional Abierta y a Distancia UNAD, 1994. Disponible en: <http://hgiobuitragov.blogspot.es/1401156926/titulo/>. Acceso en: 29 abr. 2016.

UNESCO. La educación encierra un tesoro. Informe de la Comisión Internacional sobre la Educación para el siglo XXI, presidida por J. Delors. Madrid: Santillana, 1996. 